\title{
Is there a nonadherent subtype of hypertensive patient? A latent class analysis approach
}

This article was published in the following Dove Press journal:

Patient Preference and Adherence

14 July 2010

Number of times this article has been viewed

\author{
Ranak B Trivedi' \\ Brian J Ayotte 2 \\ Carolyn T Thorpe ${ }^{3}$ \\ David Edelman ${ }^{4}$ \\ Hayden B Bosworth ${ }^{5}$ \\ 'Northwest Health Services Research \\ and Development Service Center of \\ Excellence, VA Puget Sound Health \\ Care System, Seattle, Washington; \\ ${ }^{2}$ Boston VA Health Care System, \\ Boston, Massachusetts; ${ }^{3}$ Department \\ of Population Health Sciences, \\ University of Wisconsin, Madison, \\ Wisconsin; ${ }^{4}$ Department of Medicine, \\ Duke University Medical Center, \\ Durham, North Carolina; ${ }^{5}$ Research \\ Professor, Department of Medicine, \\ Duke University Medical Center. \\ Durham, North Carolina, USA
}

Correspondence: Ranak B Trivedi

II 00 Olive Way, Suite I400,

Seattle,WA 98I0I, USA

$\mathrm{Tel}+\mathrm{I} 2067642208$

Fax + I 2067642935

Email ranak.trivedi@va.gov

\begin{abstract}
To determine subtypes of adherence, 636 hypertensive patients (48\% White, 34\% male) reported adherence to medications, diet, exercise, smoking, and home blood pressure monitoring. A latent class analysis approach was used to identify subgroups that adhere to these five self-management behaviors. Fit statistics suggested two latent classes. The first class (labeled "more adherent") included patients with greater probability of adhering to recommendations compared with the second class (labeled "less adherent") with regard to nonsmoking $(97.7 \%$ versus $76.3 \%$ ), medications ( $75.5 \%$ versus $49.5 \%$ ), diet (70.7\% versus $46.9 \%)$, exercise $(63.4 \%$ versus $27.2 \%$ ), and blood pressure monitoring (32\% versus $3.4 \%$ ). Logistic regression analyses used to characterize the two classes showed that "more adherent" participants were more likely to report full-time employment, adequate income, and better emotional and physical well-being. Results suggest the presence of a less adherent subtype of hypertensive patients. Behavioral interventions designed to improve adherence might best target these at-risk patients for greater treatment efficiency.
\end{abstract}

Keywords: adherence, hypertension, latent class analysis, self-management

\section{Introduction}

Hypertension is a major public health problem that affects 65 million Americans. ${ }^{1}$ Several trials have led to established clinical guidelines, including following medication regimens, dietary and exercise recommendations, and smoking cessation..$^{2-9}$ Adherence to these guidelines is considered necessary to optimize hypertension control. Unfortunately, hypertension control remains suboptimal, leading to an increase in the incidence of sequelae of hypertension, including heart failure and end-stage renal disease. ${ }^{10-14}$

Key to improving hypertension control is identification of people at risk for nonadherence with the various components of a hypertension regimen. Several investigations attempting to identify such individuals ${ }^{15-21}$ share one major limitation, ie, they treat adherence to each recommendation as a discrete outcome. This method ignores the potential overlap between the different behaviors, in that unhealthy behaviors tend to coexist and may have a multiplicative adverse impact on health. ${ }^{10,22}$ Only a few studies have examined the collective impact of multiple unhealthy behaviors on health outcomes. Weir et al used cluster analysis to identify subtypes of hypertensive patients based on their medication use, lifestyle behaviors, and health beliefs. ${ }^{10}$ Their results indicated the presence of four subtypes. The first group effectively managed medication and lifestyle recommendations; the second group effectively managed medications, but not lifestyle recommendations; the third group reported nonadherence to medication, diet, and exercise, but had better adherence to smoking and alcohol recommendations; and the 
fourth group reported nonadherence to all recommendations. The former two groups were found to have better health outcomes than the two latter groups. The key strength of this study was its focus on identifying the subtypes of nonadherence for hypertensive patients. This would greatly improve the ability to manage hypertension clinically by allowing greater focus on patients at risk for poor self-management. However, cluster analysis has been criticized because of the arbitrary way in which groups are created. ${ }^{23}$

Methodological limitations of cluster analysis may be avoided by using a latent class analysis (LCA) approach. LCA may be thought of as a categorical variable analog to factor analysis. ${ }^{24}$ It assumes that a categorical latent variable, or "type", causes a response on multiple observed variables. LCA has several advantages over traditional cluster analysis. LCA allows model fit criteria and rigorous statistical testing, whereas cluster analysis employs a relatively arbitrary method of clustering. ${ }^{23,25}$ An additional advantage is that LCA can be used with categorical data. Because many hypertension recommendations exist on a dichotomy (eg, stopping smoking), this can be an important methodology to employ with adherence behaviors. LCA may provide an important methodology for identifying subtypes of nonadherence, while circumventing limitations in the methodologies that have been employed in the literature.

The purpose of this study was to determine the presence of underlying adherence subtypes in hypertensive patients using an LCA approach. We were interested in identifying subtypes of hypertensive patients based on their self-management behaviors. Secondary aims were to characterize the subtypes based on a variety of demographic and psychosocial variables, ie, age, race, gender, financial situation, employment, quality of life, social support, stress, and attitudes towards hypertension. Characterization of the nonadherent subtype would potentially identify specific psychosocial factors worth targeting in adherence-improving interventions. Finally, we examined the association of adherence subtypes on blood pressure (BP) levels.

\section{Methods}

\section{Participants}

Six hundred thirty-six hypertensive patients were recruited from two Duke University Medical Center primary care clinics to participate in the Take Control of Your Blood Pressure study. ${ }^{26}$ Briefly, this study tested two interventions (tailored behavioral intervention and BP self-monitoring) in a sample of hypertensive patients. ${ }^{27}$ The present study represents secondary, cross-sectional analyses of the baseline data from this trial. Participants were $66 \%$ female, and were evenly split between White and African American races (48.4\% and $49 \%$, respectively; $2.6 \%$ were classified as "other" for these analyses). All procedures were approved by the Institutional Review Board of Duke University Medical Center.

\section{Inclusion and exclusion criteria}

Patients were included if they had documented hypertension according to medical records (ICD-9 codes 401.9, 401.0, 401.1), if they had been enrolled in one of the two primary care clinics for at least a year, and if they were using a hypertensive medication, eg, an angiotensin-converting enzyme inhibitor or a beta-blocker. Patients were excluded if they were not on a BP medication; a family member was already enrolled in the study; they did not live in an eight-county catchment area; were receiving kidney dialysis; were pregnant or were planning to be pregnant; had an arm circumference greater than 17 inches and wrist circumference greater than 8.5 inches; had been hospitalized for a stroke, myocardial infarction, or coronary artery revascularization within the previous three months; had been diagnosed with metastatic cancer or dementia; resided in a nursing home or received home health care; did not speak or understand English; were enrolled in another hypertension study; were not receiving the majority of their healthcare through Duke University; had severely impaired hearing or speech; and/or had a history of organ transplantation. Using this method, 7646 potentially eligible patients were identified using medical records; 1325 were deemed eligible per the above criteria and were contacted. Six hundred and thirty-six patients (48\% of the 1325) agreed to participate and were enrolled. For more details regarding the study design, refer to Bosworth et al. ${ }^{26}$

\section{Blood pressure measurement}

Patients underwent BP screening involving two successive digitally-derived BP values taken by study staff. These were averaged to determine baseline systolic and diastolic BP (SBP and DBP, respectively). We were further interested in examining BP control. Using JNC 7 guidelines, SBP control was defined as $\mathrm{SBP} \leq 140 \mathrm{mmHg}$ and $\mathrm{DBP}$ control as $\mathrm{DBP} \leq 90 \mathrm{mmHg}$.

\section{Adherence measures}

A variety of measures were used to assess adherence to medications, diet, exercise, smoking, and home BP monitoring. All adherence measures were dichotomized. Exercise, 
medication adherence, and smoking recommendations were dichotomized at the point which best represented contemporary public health recommendations. Details regarding the cutoffs are provided below.

Medication adherence was assessed using the Self-reported Medication Taking Scale. ${ }^{28}$ This four-item measure assesses medication-taking behavior. Respondents rate whether they forget to take medications, are careless about taking their medications, or stop taking their medications based on whether they feel better or worse. Items are rated on a four-point scale, from "strongly agree" to "strongly disagree". In the current study, the internal consistency as measured by Cronbach's alpha was 0.85 . As in prior research, participants who endorsed at least one item by answering either "strongly agree" or "agree" were considered nonadherent to medications.

To define adherence to exercise recommendations, patients were asked: "On average, how much time per week do you spend on aerobic or body movement activities, such as brisk walking, jogging, or running, that elevates your heart rate for 20 minutes and makes you sweat/perspire?" Five answer options ranged from "never" to "more than four hours/week". Participants were considered to be nonadherent to exercise if they reported exercising $<2$ hours/week, consistent with current recommendations. ${ }^{29}$

Participants were asked: "On a scale of 1 to 10 , with 1 being not at all hard and 10 being extremely hard, please rate how hard it is for you to follow recommendations to improve your blood pressure regarding diet". Participants were considered nonadherent to dietary recommendations if they rated their difficulty with dietary recommendations $\geq 5$ on the 10-point scale.

Smoking status was determined by a single yes/no item asking whether participants were current cigarette smokers. Participants were considered nonadherent to smoking recommendations if they reported that they currently smoked.

Patients were asked if they owned a home BP monitor and, if so, how often they used the monitor. Answers ranged from "never" to "frequently". Patients were considered nonadherent if they answered "never".

\section{Psychosocial measures}

Because we were also interested in characterizing the patients, we collected data on a variety of demographic variables and psychosocial constructs. A demographic questionnaire was administered to determine participants' age, gender, ethnicity, marital status, education, employment status, and financial situation. Data were reduced for the following variables: race (White versus African American), marital status (married versus unmarried), education (equal to or greater than high school versus less than high school), and employment status (employed versus unemployed). Participants were asked about the number of people residing with them because cohabitation has been shown to have a positive effect on adherence. ${ }^{30}$

Two subscales of the MOS Short Form 12 (SF-12) were used to measure emotional well-being and physical well-being. ${ }^{31,32}$ Emotional well-being was measured using the Mental Component Summary Scale (MCS), a five-item subscale that measures general mental health and its impact on daily functioning. Physical well-being was measures using the Physical Component Summary Scale (PCS), a five-item subscale that measures general physical health and its impact on daily functioning. The mean of each subscale is 50 , the standard deviation (SD) is 10 , and the reliability ranges from 0.77-0.97..$^{31,32}$ To allow for clinical interpretation, MCS and PCS scores were divided by 10 , which is $1 \mathrm{SD}$. These scores were subsequently used in regression models.

Social support was measured in two ways. First, instrumental support was measured by asking participants: "If needed, is there someone who could help you with tasks such as taking you to the doctor, fixing lunch, or home repairs?" Second, emotional support was measured by asking participants: "Do you have someone you feel close to, someone you can trust and confide in?" The instrumental and emotional support items were answered either "yes" or "no". Participants were further asked how much contact they had with the person in whom they could trust or confide. This was rated on a four-point Likert scale ranging from "no contact" to "a lot of contact".

Participants were asked how often in the past month they had experienced stress. Answers were rated on a five-point Likert scale ranging from "never" to "very often".

Attitudes towards hypertension were assessed by asking participants to what extent they believed hypertension was a serious condition. Answers were rated on a four-point Likert scale, from "very serious" to "not at all serious". They were also asked how worried they were about hypertension. Answers were rated on a 10-point scale, from "definitely not worried" to "extremely worried".

\section{Data analysis}

All analyses were conducted using Mplus 4.1 (Muthen and Muthen, Los Angeles, CA). The analytical strategy in LCA involves identifying the fewest number of classes that explain adherence across the five recommended self-management behaviors. The optimal number of classes was determined by progressively increasing the number of classes and testing each subsequent model. Because there remains debate on 
the best way to determine number of classes, a combination of Bayesian information criterion (BIC) and parametric likelihood ratio tests with bootstrapped values (LRT) was used to determine model fit. The BIC aims to balance model fit with model parsimony such that lower scores represent better model fit. ${ }^{25}$ The LRT compares progressive iterations of the more parsimonious models ( $k-1$ classes) against models with greater number of classes ( $k$ classes). To establish the number of classes using LRT, the models with the fewest number of classes where $P<0.05$ were accepted. ${ }^{33}$ Using this combined strategy, we assigned each participant to a permanent class, based on their highest posterior class membership probability of being in each class.

After establishing class membership, logistic regression analyses were conducted to examine the odds of being a member of the classes given responses to the psychosocial and demographic questions, and SBP and DBP levels. The class less likely to be adherent was used as the referent. Significance for regression analyses was set at $P<0.05$.

\section{Results}

The sample of 636 patients with hypertension $(66 \%$ female, 49\% African American) is described in Table 1. At baseline, the mean SBP was $125.0 \mathrm{mmHg}$ and mean DBP was $71.3 \mathrm{mmHg}$. Approximately $84 \%$ of participants reported that they were nonsmokers, $58 \%$ reported adherence to medication, $55 \%$ reported adherence to dietary recommendations, $40 \%$ reported adherence to exercise recommendations, and 13\% reported current home BP monitor use.

To determine the presence of latent classes for adherence behavior, 1, 2, and 3 class models were tested. As

Table I Baseline patient characteristics

\begin{tabular}{ll}
\hline $\mathbf{n}$ & $\mathbf{6 3 6}$ \\
\hline Age (mean \pm SD) & $61.25 \pm 12.32$ (range 25-92) \\
Gender, $\mathrm{n}(\%)$ & 420 female $(66 \%)$ \\
Race & $48.4 \%$ White, 49.0\% African American \\
Baseline SBP, mmHg & $125.0 \pm 17.7$ \\
Baseline DBP, mmHg & $71.3 \pm 10.8$ \\
Current smokers, $\mathrm{n}(\%)$ & $130(16.4 \%)$ \\
Adherence to dietary & $346(55.1 \%)$ \\
recommendations, $\mathrm{n}(\%)$ & \\
Adherence to exercise & $252(39.62 \%)$ \\
recommendations, $\mathrm{n}(\%)$ & \\
Adherence to medication, $\mathrm{n}(\%)$ & $371(58.4 \%)$ \\
BP monitor use, $\mathrm{n}(\%)$ & $84(13.2 \%)$ \\
\hline
\end{tabular}

Abbreviations: BP, blood pressure; SBP, systolic blood pressure; DBP, diastolic blood pressure; SD, standard deviation.
Table 2 Fit statistics of the latent class analysis models

\begin{tabular}{llll}
\hline Classes & Log-likelihood & BIC & $\begin{array}{l}\text { Bootstrapped } p \text { value } \\
\text { from LRT for } k \text { - I classes }\end{array}$ \\
\hline 1 & -1823.97 & 3675.07 & \\
2 & -1796.09 & 3663.19 & $P<0.001$ \\
3 & -1791.82 & 3692.38 & $P=0.15$ \\
\hline
\end{tabular}

Abbreviations: BIC, Bayesian information criterion; LRT, likelihood ratio test.

shown in Table 2, a 2-class model was superior to both 1 - and 3-class models by virtue of lower BIC and $P<$ 0.05 associated with the LRT 2 versus 3 -class model. Class 1 consisted of $34.4 \%(n=219)$ of the sample. These participants were consistently more likely to report adherence to medications, nonsmoking, diet, exercise, and home BP recommendations. Class 2 consisted of the remainder $65.6 \%(n=417)$ of the sample. Compared with participants assigned to Class 1, Class 2 participants had a lower probability of reporting adherence to the five behaviors. Based on this, Class 1 was classified as "more adherent" and Class 2 was classified as "less adherent". Conditional probabilities are provided in Table 3 and represented in Figure 1. As can be seen in Figure 1, the patterns of adherence were similar in both classes, with most participants reporting current nonsmoking status, and fewest participants reporting home BP monitor use.

Next, logistic regression analyses were conducted to compare characteristics of the "more adherent" class to the "less adherent" class (Table 4). "More adherent" patients were more likely to report full-time employment (odds ratio [OR] $0.79,95 \%$ confidence interval $[\mathrm{CI}]: 0.64-0.96)$ and fewer problems with finances (OR 0.77, 95\% CI: 0.62-0.96). For every 10-point increase in emotional well-being, represented by MCS scores, the odds of a patient being "more adherent" increased by 24\% (OR 1.24, 95\% CI: 1.01-1.53). Similarly, for every 10-point increase in physical well-being,

Table 3 Conditional probabilities of class membership in the two-class mode

\begin{tabular}{lll}
\hline & $\begin{array}{l}\text { Class I: “More } \\
\text { adherent" }\end{array}$ & $\begin{array}{l}\text { Class 2: “Less } \\
\text { adherent" }\end{array}$ \\
\hline $\begin{array}{l}\text { Takes medications as prescribed } \\
\text { Does not have difficulty adhering } \\
\text { to dietary recommendations }\end{array}$ & 0.755 & 0.495 \\
$\begin{array}{l}\text { Exercises at least } 30 \mathrm{~min} / \text { day, } \\
\text { most days/week }\end{array}$ & 0.634 & 0.469 \\
$\begin{array}{l}\text { Does not currently } \\
\text { smoke cigarettes }\end{array}$ & 0.977 & 0.272 \\
$\begin{array}{l}\text { Uses home blood } \\
\text { pressure monitor }\end{array}$ & 0.320 & 0.763 \\
\hline
\end{tabular}




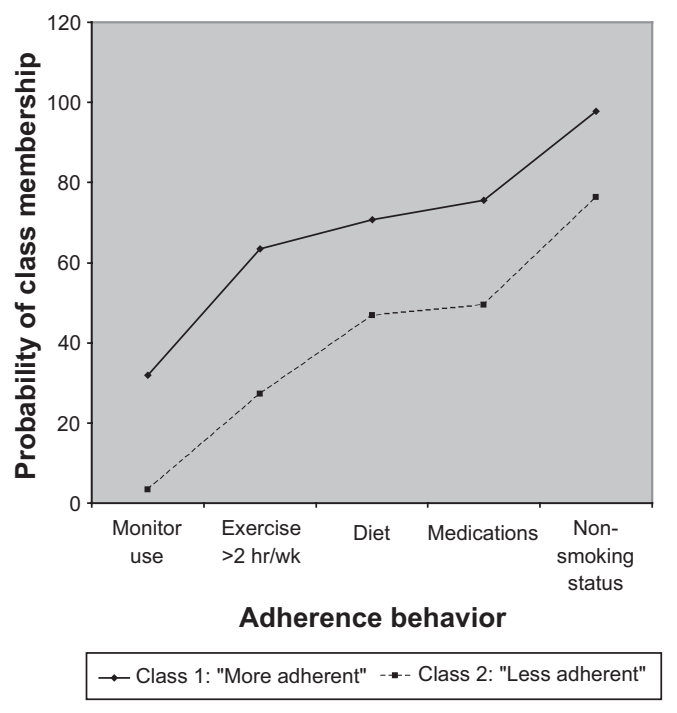

Figure I Conditional probabilities of being adherent to the five recommendations, given class membership.

represented by PCS scores, the patients' odds of being "more adherent" increased by 30\% (OR 1.30, 95\% CI: 1.06-1.59). Age, race, marital status, gender, or education levels did not appear to impact class membership. No differences in SBP, DBP, or BP control were found.

\section{Discussion}

This study sought to demonstrate the presence of subtypes of hypertensive patients who are either more or less adherent across several hypertension-related self-management behaviors. Two distinct subtypes of hypertensive patients were identified based on adherence to five self-management recommendations. Previous investigations have used LCA to show that health behaviors may be multidimensional. ${ }^{34}$ However, to our knowledge, this is the first investigation to use LCA and demonstrated the presence of subtypes of adherence in hypertensive patients.

The next goal was to characterize the two classes. Previous investigations have focused on many factors that might impact nonadherence, with mixed results. Age, gender, and personality traits have all been examined as potential predictors or correlates of adherence, with weak and inconsistent results. ${ }^{20,35}$ Race has been more consistently predictive of adherence, with Whites demonstrating better adherence than African Americans. ${ }^{36}$ It should be noted that the vast majority of these studies have focused on medication adherence, with little attention to other self-management behaviors. In this study, age, race, marital status, and gender were not related to class membership. These results may provide further
Table 4 Odds ratio of being a member of the "more adherent" class

\begin{tabular}{lll}
\hline Variable & OR & 95\% CI \\
\hline Age (per I0-year increase) & 1.16 & $0.97-1.40$ \\
Race (White versus Non-White) & 1.34 & $0.86-2.12$ \\
Married (married versus unmarried) & 1.31 & $0.80-2.06$ \\
Gender (female versus male) & 0.77 & $0.48-1.24$ \\
Education (high school versus no & 1.12 & $0.97-1.30$ \\
high school) & & \\
SBP (per 5 mmHg increase) & 1.04 & $0.98-1.11$ \\
DBP (per 5 mmHg increase) & 0.96 & $0.87-1.07$ \\
SBP control & 0.84 & $0.51-1.40$ \\
DBP control & 0.77 & $0.48-1.23$ \\
SF-I2 Physical Component Summary & $1.30^{*}$ & $1.06-1.59$ \\
Score (per I0-point increase) & & \\
SF-I2 Mental Component Summary & $1.24^{*}$ & $1.01-1.53$ \\
Score (per I0-point increase) & & \\
Number of cohabitants & 0.88 & $0.73-1.05$ \\
Instrumental support & 1.11 & $0.45-2.73$ \\
Amount of contact with social support & 1.05 & $0.70-1.58$ \\
Perceived stress & 0.89 & $0.72-1.09$ \\
Employment status & $0.79^{*}$ & $0.64-0.96$ \\
Financial situation & $0.77^{*}$ & $0.62-0.96$ \\
Perceived seriousness of hypertension & 0.88 & $0.59-1.33$ \\
Worry about hypertension & 0.96 & $0.88-1.06$ \\
Availability of leisure time & 1.06 & $0.91-1.24$ \\
\hline Abbrevitions: OR, & &
\end{tabular}

Abbreviations: $\mathrm{OR}$, odds ratio; $\mathrm{Cl}$, confidence interval; DBP, diastolic blood pressure; SBP, systolic blood pressure. ${ }^{*} p<0.05$.

insight into the role of these traditional demographic variables in adherence as a latent class and might explain the contradictory results that have been documented in the literature when adherence behavior is examined individually.

Being more adherent was related to better physical and emotional well-being. We have previously reported that emotional well-being is related to adherence to diet and exercise and smoking recommendations. ${ }^{20}$ These findings provide further support that lower emotional well-being, even if it does not meet clinical significance, may adversely influence adherence to all recommendations. This underscores the importance of assessing emotional well-being in primary care settings.

Results suggest that lack of employment and financial difficulties may be important barriers to optimally managing hypertension. Financial constraints may limit patients' ability to purchase medications. In addition, the necessity of working extra hours for income may limit the time patients can devote to exercise or limit access to resources such as gyms, personal trainers, or dietitians. Improving access to health care, which is a goal of "Healthy People 2010", may be critical in reducing the burden on both the individual and society as a whole. 
Contrary to our hypothesis, adherence subtypes were not related to BP levels or BP control. The relationship between adherence and outcomes is complex, and may be influenced by dose, efficacy of treatment, response rates, and understanding of the disease. ${ }^{37}$ Past investigations suggest that the impact of adherence on disease-specific medical outcomes may be limited even when the impact on other indices, such as emotional well-being, may be strong. ${ }^{10,37,38}$ Several explanations of our results may be posited. First, the sample in this study consisted of patients with well-controlled hypertension, as can be seen from the mean and SD of the sample BP values. Second, the sample reported high adherence to medications, perhaps the single most important factor in controlling hypertension in the short term. Third, our ability to detect causality may be hampered because adherence and BP were measured concurrently in this cross-sectional study. Given all these factors, the lack of relationship between the adherence classes and BP may illustrate that even within a highly adherent, well-controlled group of hypertensive patients, adherence behaviors may covary. Studies interested in understanding adherence should recognize that individually treating each behavior as unique ignores this covariance and likely inflates the Type I error. It is possible that previous studies linking individual behaviors to BP may not be as robust, given the potential of Type I error that results from multiple comparisons. Because this study utilized a novel approach to examining adherence, rigorous investigations are needed to examine the impact of nonadherence to multiple behaviors on BP levels in studies specifically designed to answer this question.

Our findings support the notion that the focus of adherence interventions can be potentially broadened to target multiple behaviors. Historically, interventions aimed at improving adherence target behaviors individually (eg, smoking cessation and exercise regimens). Inevitably, interventions targeting one behavior will be limited in their impact. ${ }^{39}$ These interventions may also be time-consuming and costly, and may not reflect the relationship between various adherence behaviors. In reality, making changes to one health behavior may serve as a catalyst to making other lifestyle changes. ${ }^{40}$ Based on such findings, recent interventions have focused on multiple health behavior changes ${ }^{41}$ ie, interventions that may impact multiple behaviors simultaneously. These interventions may demonstrate an overall benefit even though their impact on an isolated behavior may be too small to detect if examined individually.
This study has some limitations. First, the crosssectional nature of the data limits our ability to examine the predictive validity of the two subtypes. It is not clear whether these adherent/nonadherent subtypes would impact hypertension and other related outcomes prospectively. Second, the cross-sectional nature of the data does not allow us to determine predictors of class membership. Third, the use of multiple single-item measures raises questions regarding reliability and validity. Results of these analyses should be considered hypothesesgenerating, given the potential psychometric difficulties of single-item measures. Fourth, our population was both relatively well-controlled and relatively adherent. Predictors of adherence behavior may not be the same in a more nonadherent population.

Despite these limitations, this study makes an important contribution to the extensive adherence literature in hypertensive patients. It proposes a unique way of characterizing hypertensive patients that may be both methodologically sound and clinically relevant. It also highlights the complexity inherent in obtaining adequate hypertension control. Further investigations are necessary to replicate these results, especially given the previous literature negating the presence of an "adherent personality". Future investigations should focus on the predictive validity of the two subtypes as well as predictors of class membership. If replicated, these results provide support for designing interventions aimed at multiple health behaviors in the millions of Americans currently treated for hypertension.

\section{Acknowledgments}

This study was supported by NHLBI Grant R01 HL070713, a Pfizer Health Communication Initiative Award, and an American Heart Association Established Investigator Award, awarded to HBB, the principal investigator, who has full access to all of the data in the study and takes responsibility for the integrity of the data and its analysis. Results were initially presented at the 67th Annual Meeting of the American Psychosomatic Society. This work was supported in part by the facilities provided by the VA Puget Sound Health Care System where RBT is a core investigator. The views expressed in this article are those of the authors and do not necessarily reflect the position or policy of the Department of Veterans Affairs or the US government. We would like to thank Dr Joshua Thorpe for his suggestions regarding presentation of the analytic approach and results. 


\section{Disclosure}

HBB received funds from the Pfizer Health Communication Initiative Award, but the organization was not involved in the data collection, analyses, or interpretation of the results.

\section{References}

1. Fields LE, Burt VL, Cutler JA, Hughes J, Roccella EJ, Sorlie P. The burden of adult hypertension in the United States 1999 to 2000: A rising tide. Hypertension. 2004;44(4):398-404.

2. The ALLHAT Officers and Coordinators for the ALLHAT Collaborative Research Group. Major outcomes in high-risk hypertensive patients randomized to angiotensin-converting enzyme inhibitor or calcium channel blocker vs diuretic: The Antihypertensive and Lipid-Lowering Treatment to Prevent Heart Attack Trial (ALLHAT). JAMA. 2002; 288(23):2981-2997.

3. Psaty BM, Lumley T, Furberg CD, et al. Health outcomes associated with various antihypertensive therapies used as first-line agents: A network meta-analysis. JAMA. 2003;289(19):2534-2544.

4. Chobanian AV, Bakris GL, Black HR, et al. The Seventh Report of the Joint National Committee on Prevention, Detection, Evaluation, and Treatment of High Blood Pressure: The JNC 7 report. JAMA. 2003; 289(19):2560-2572.

5. Elmer PJ, Grimm R Jr, Laing B, et al. Lifestyle intervention: Results of the Treatment of Mild Hypertension Study (TOMHS). Prev Med. 1995; 24(4):378-388.

6. Lichtenstein AH, Appel LJ, Brands M, et al. Diet and lifestyle recommendations revision 2006: A scientific statement from the American Heart Association Nutrition Committee. Circulation. 2006; 114(1):82-96.

7. Miller ER 3rd, Erlinger TP, Young DR, et al. Results of the Diet, Exercise, and Weight Loss Intervention Trial (DEW-IT). Hypertension. 2002;40(5):612-618.

8. Sacks FM, Svetkey LP, Vollmer WM, et al. Effects on blood pressure of reduced dietary sodium and the Dietary Approaches to Stop Hypertension (DASH) diet. DASH-Sodium Collaborative Research Group. N Engl J Med. 2001;344(1):3-10.

9. Whelton PK, Appel LJ, Espeland MA, et al. Sodium reduction and weight loss in the treatment of hypertension in older persons: A randomized controlled trial of nonpharmacologic interventions in the elderly (TONE). TONE Collaborative Research Group. JAMA 1998;279(11):839-846.

10. Weir MR, Maibach EW, Bakris GL, et al. Implications of a healthy lifestyle and medication analysis for improving hypertension control. Arch Intern Med. 2000;160(4):481-490.

11. He J, Muntner P, Chen J, Roccella EJ, Streiffer RH, Whelton PK. Factors associated with hypertension control in the general population of the United States. Arch Intern Med. 2002;162(9):1051-1058.

12. Banegas JR, Guallar-Castillon P, Rodriguez-Artalejo F, Graciani A, Lopez-Garcia E, Ruilope LM. Association between awareness, treatment, and control of hypertension, and quality of life among older adults in Spain. Am J Hypertens. 2006;19(7): 686-693.

13. Burke GL, Sprafka JM, Folsom AR, Luepker RV, Norsted SW, Blackburn H. Trends in CHD mortality, morbidity and risk factor levels from 1960 to 1986: The Minnesota Heart Survey. Int J Epidemiol. 1989;18(3 Suppl 1):S73-S81.

14. White WB. Improving blood pressure control and clinical outcomes through initial use of combination therapy in Stage 2 hypertension. Blood Press Monit. 2008;13(2):123-129.

15. Kim MT, Han HR, Hill MN, Rose L, Roary M. Depression, substance use, adherence behaviors, and blood pressure in urban hypertensive black men. Ann Behav Med. 2003;26(1):24-31.
16. Morris AB, Li J, Kroenke K, Bruner-England TE, Young JM, Murray MD. Factors associated with drug adherence and blood pressure control in patients with hypertension. Pharmacotherapy. 2006;26(4): 483-492.

17. Bosworth HB, Dudley T, Olsen MK, et al. Racial differences in blood pressure control: Potential explanatory factors. Am J Med. 2006; 119(1):70 e79-15.

18. Patel RP, Taylor SD. Factors affecting medication adherence in hypertensive patients. Ann Pharmacother. 2002;36(1):40-45.

19. Fuertes JN, Mislowack A, Bennett J, et al. The physician-patient working alliance. Patient Educ Couns. 2007;66(1):29-36.

20. Trivedi RB, Ayotte B, Edelman D, Bosworth HB. The association of emotional well-being and marital status with treatment adherence among patients with hypertension. J Behav Med. 2008;31(6):489-497.

21. Wang PS, Bohn RL, Knight E, Glynn RJ, Mogun H, Avorn J. Noncompliance with antihypertensive medications: The impact of depressive symptoms and psychosocial factors. $J$ Gen Intern Med. 2002;17(7):504-511.

22. Fine LJ, Philogene GS, Gramling R, Coups EJ, Sinha S. Prevalence of multiple chronic disease risk factors. 2001 National Health Interview Survey. Am J Prev Med. 2004;27(2 Suppl):18-24.

23. Magidson J, Vermunt JK. Latent class models for clustering: A comparison with K-means. CJMR. 2002;20:36-43.

24. McCutcheon AL. Latent Class Analysis. Vol 64. Newbury Park, CA: Sage Publications; 1987.

25. Beeber AS, Thorpe JM, Clipp EC. Community-based service use by elders with dementia and their caregivers: A latent class analysis. Nurs Res. 2008;57(5):312-321.

26. Bosworth HB, Olsen MK, Dudley T, et al. The Take Control of Your Blood pressure (TCYB) study: Study design and methodology. Contemp Clin Trials. 2007;28(1):33-47.

27. Bosworth HB, Olsen MK, Grubber JM, et al. Two self-management interventions to improve hypertension control: A randomized trial. Ann Intern Med. 2009;151(10):687-695.

28. Morisky DE, Green LW, Levine DM. Concurrent and predictive validity of a self-reported measure of medication adherence. Med Care. 1986;24(1):67-74

29. Haskell WL, Lee IM, Pate RR, et al. Physical activity and public health: Updated recommendation for adults from the American College of Sports Medicine and the American Heart Association. Med Sci Sports Exerc. 2007;39(8):1423-1434.

30. DiMatteo MR. Social support and patient adherence to medical treatment: A meta-analysis. Health Psychol. 2004;23(2):207-218.

31. Ware J Jr, Kosinski M, Keller SD. A 12-Item Short-Form Health Survey: Construction of scales and preliminary tests of reliability and validity. Med Care. 1996;34(3):220-233.

32. Gandek B, Ware JE, Aaronson NK, et al. Cross-validation of item selection and scoring for the SF-12 Health Survey in nine countries: Results from the IQOLA Project. International Quality of Life Assessment. J Clin Epidemiol. 1998;51(11):1171-1178.

33. Vermunt JK, Magidson J. Latent GOLD User's Manual. Boston, MA Statistical Innovations, Inc.; 2000.

34. Ingledew DK, Hardy L, Cooper CL. Latent class analysis applied to health behaviours. Pers Individ Dif. 1995;19(1):13-20.

35. Miller NH, Hill M, Kottke T, Ockene IS. The multilevel compliance challenge: Recommendations for a call to action: A statement for healthcare professionals. Circulation. 1997;95(4): 1085-1090.

36. Borzecki AM, Oliveria SA, Berlowitz DR. Barriers to hypertension control. Am Heart J. 2005;149(5):785-794.

37. DiMatteo MR, Giordani PJ, Lepper HS, Croghan TW. Patient adherence and medical treatment outcomes: A meta-analysis. Med Care. 2002;40(9):794-811.

38. Natarajan S, Santa Ana EJ, Liao Y, Lipsitz SR, McGee DL. Effect of treatment and adherence on ethnic differences in blood pressure control among adults with hypertension. Ann Epidemiol. 2009; 19(3):172-179. 
39. Hayes SC, Barlow DH, Nelson-Gray RO. The Scientist Practitioner: Research and Accountability in the Age of Managed Care. 2nd ed. Boston, MA: Allyn and Bacon; 1999.

40. Nagaya T, Yoshida H, Takahashi H, Kawai M. Cigarette smoking weakens exercise habits in healthy men. Nic Tob Res. 2007;9(10):1027-1032.
41. Prochaska JJ, Spring B, Nigg CR. Multiple health behavior change research: An introduction and overview. Prev Med. 2008;46(3): $181-188$.

\section{Publish your work in this journal}

Patient Preference and Adherence is an international, peer-reviewed, open access journal that focusing on the growing importance of patient preference and adherence throughout the therapeutic continuum. Patient satisfaction, acceptability, quality of life, compliance, persistence and their role in developing new therapeutic modalities and compounds to optimize clinical outcomes for existing disease states are major areas of interest. This journal has been accepted for indexing on PubMed Central. The manuscript management system is completely online and includes a very quick and fair peer-review system. Visit http://www.dovepress.com/ testimonials.php to read real quotes from published authors.

Submit your manuscript here: http://www.dovepress.com/patient-preference-and-adherence-journal 